\title{
Semaphorins as signals for cell repulsion and invasion
}

\author{
Yoshio Goshima, ${ }^{1,3}$ Takaaki Ito, ${ }^{2}$ Yukio Sasaki, ${ }^{1}$ and Fumio Nakamura ${ }^{1}$ \\ ${ }^{1}$ Department of Molecular Pharmacology and Neurobiology, and \\ ${ }^{2}$ Department of Pathology, Yokohama City University School of Medicine, Yokohama, Japan \\ ${ }^{3}$ Core Research for Evolutional Science and Technology (CREST), Japan Science and Technology Corp., Saitama, Japan \\ Address correspondence to: Yoshio Goshima, Department of Molecular Pharmacology and Neurobiology, \\ Yokohama City University School of Medicine, 3-9 Fuku-ura, Kanazawa-ku, Yokohama 236-0004, Yokohama, Japan. \\ Phone: 81-45-787-2593; Fax: 81-45-785-3645; E-mail: goshima@med.yokohama-cu.ac.jp.
}

J. Clin. Invest. 109:993-998 (2002). DOI:10.1172/JCI200215467.

Semaphorins are a large family of secreted and transmembrane signaling proteins that regulate axonal guidance in the developing nervous system. Recent studies suggest that semaphorin receptors also act in such diverse processes as lymphocyte activation, control of vascular endothelial cell motility, and lung branching morphogenesis. Here, we describe the current knowledge of the intracellular signaling pathways employed by these molecules, focusing on several candidate molecules that help reorganize the cytoskeleton or alter patterns of intracellular transport during neuronal outgrowth. We then consider other cellular events, occurring outside of the nervous system, that are mediated by these semaphorins, their receptors, and associated molecules.

\section{Semaphorins in axon guidance}

The shapes of neurons are the most varied and elaborate of all cells. The distal tip of growing neuritic processes, the growth cone, distinguishes between multiple pathways and synaptic targets during development and regeneration. As in other cell types, morphological changes and motility in neurons are closely related to reorganization of cytoskeletal proteins. The growth cone's high sensitivity to small gradients of attractive and repulsive signals in the environment, and its ability to rapidly rearrange its morphology - and that of its associated axon - in response to even a single filopodial contact, suggest that it contains a powerful signal amplification system.

The semaphorins are among the best-studied axonal guidance molecules and appear to play key roles in these cellular events. To date, more than 20 different semaphorins have been identified (1). They fall into eight groups on the basis of domain organization and species of origin, but all semaphorins contain a conserved, 500-amino-acid-length "Sema" domain at their amino-terminus. This domain is also present in the plexin (Plex) family and in the Met oncoprotein. Sema3A, a prototypical class 3 , secreted semaphorin, is a potent inhibitor of axonal outgrowth from a specific subset of neurons, including spinal motoneurons and neurons in the embryonic dorsal root ganglion (DRG) and sympathetic ganglion (2-5). The binding of Sema3A to DRG growth cones rapidly induces actin depolymerization and growth cone collapse, a cellular response associated with the chemorepulsion of neurites. However, semaphorins can provide both repulsive and attractive cues. For instance, in cortical neurons, where Sema3A provides a repulsive signal, Sema3C acts as an attractive guidance signal (see ref. 5 for review). Moreover, the effect of semaphorin signaling can be altered by changes in neuronal physiology, as seen in Xenopus spinal neurons, where Sema3A-induced repulsion can be changed to attraction by an analogue of guanosine $3^{\prime}, 5^{\prime}$-monophosphate.

\section{Functional receptor complexes for semaphorins}

Cell surface receptors for semaphorins have been identified as the members of two distinct transmembrane receptor families, neuropilin (NP) and Plex. NP-1 and NP-2 were identified by their ability to bind the secreted class 3 semaphorins $(2,3,6)$. In particular, Sema3A signaling is mediated by a receptor complex involving NP-1, whereas Sema3F signaling occurs through NP-2 $(2,3,5)$, and heteromeric NP-1/NP-2 complexes are thought to serve as functional receptors for Sema3C.

Semaphorin receptors belonging to the more recently identified Plex family carry a conserved Sema domain, suggesting that these receptors and their ligands derive from a common evolutionary ancestor with homophilic binding properties $(5,7)$. Among invertebrates, semaphorins and their receptors are perhaps best studied in Drosophila, where PlexA and Sema1a have been shown to interact genetically. Biochemical studies show that the Drosophila Sema-1a and Sema-1b proteins bind with high affinity to Plex-A (5). Vertebrates express at least nine Plex family members, including the functional receptors for classes 1,4 , and 7 transmembrane semaphorins $(8,9)$. Plex-C1, also known as VESPR, has been identified as a cellular receptor for a class 5 semaphorin expressed by poxvirus. At least two vertebrate plexins act directly as semaphorin receptors (9). Plex-B1 binds Sema4D (CD100), and Plex-C1 
(VESPR) binds Sema7A, consistent with the observation that Sema7A is the host semaphorin that shares the greatest sequence similarity to viral semaphorins. The class 3 semaphorins, including Sema3A, Sema3C, and Sema3F, bind directly to NPs but do not bind to Plex family members. Nonetheless, NP-1 and Plex-A1 form a complex with enhanced affinity for Sema3A (8, 9). Plex proteins are now thought to constitute a signaling moiety in a receptor complex for the class $3 \mathrm{sem}$ aphorins, where NPs provide the ligand-binding sites. Recent data suggest that Plex-A1 is subject to autoinhibition mediated by its intrinsic Sema domain (10). Sema-deleted Plex-A1 is constitutively active, producing cell contraction, growth cone collapse, and inhibition of neurite outgrowth. This Sema domain of Plex-A1 physically associates with the remainder of the Plex-A1 ectodomain and can act in trans to reverse constitutive activation. Both the Sema portion and the remainder of the ectodomain of Plex-A1 associate with NP-1 in a Sema3A-independent fashion. Hence, it appears that Plex-A1 is autoinhibited by its Sema domain but that Sema3A/NP-1 releases this inhibition.

\section{Semaphorin-induced intracellular signaling}

Although the molecular mechanisms by which the semaphorins exert their effects remain to be clarified, several intracellular molecules, including the small GTPase Rac1, the collapsin (Sema3A) response mediator protein (CRMP), and Lin-11, Isl-1, and Mec-3 (LIM) kinase, have been implicated as mediators of semaphorin signaling. Candidate mediators have been found by searching for intracellular molecules that interact with the cytoplasmic domain of transmembrane semaphorins, NPs, or Plex proteins. One such protein, NIP, interacts with the NP-1 cytoplasmic domain, as was originally shown using yeast twohybrid screening (11). In addition, NIP has been shown to be identical to GIPC, a protein that binds the heteromeric $\mathrm{G}$ protein $\mathrm{G} \alpha_{\mathrm{i} 3}-\mathrm{RGS}$, and the same protein was previously identified as SEMCAP-1, which binds the cytoplasmic domain of Sema4C, a transmembrane semaphorin. Based on these various functions, it seems possible that NIP serves to regulate the efficacy of a Sema3A receptor complex by inducing NP-1 clustering. Alternatively, it has been suggested that this protein provides a link between Sema3A- and $\mathrm{G}$ protein-signaling cascades. However, it is interesting to note that the intracellular region of NP-1, to which NIP binds, is not essential for Sema3A-induced growth cone collapse (5).

Growth cone collapse is crucial for the formation of normal patterns of innervation by developing neurons. This process is accompanied by a rapid reorganization of the actin filaments normally present in lamellipodia and filopodia, suggesting that growth cone NP-1/Plex complexes interact with signaling molecules to regulate actin polymerization or crosslinking. The Rho family of monomeric GTPases includes Rho, Rac, and Cdc42, three well-known regulators of the actin cytoskeleton of non-neuronal cells. In DRG neurons, introduction of a dominant

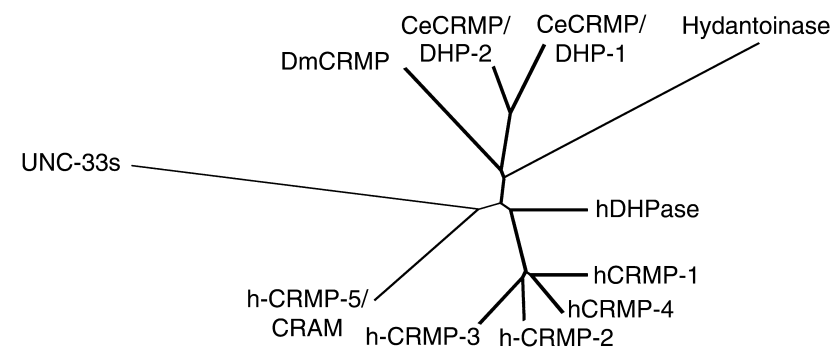

Figure 1

A phylogenic tree, showing the relationships between the vertebrate and invertebrate homologs of the CRMP, including the dihydropyrimidinase (DHPs) and UNC-33. Ce, C. elegans; Dm, Drosophila melanogaster.

negative Rac (N17) mutant blocks Sema3A-mediated growth cone collapse (12), suggesting that Rac1 acts as a downstream molecule of an NP-1/Plex receptor. Indeed, Plex-B1 binds directly to activated Rac1 (13, 14), and Plex proteins can modulate the activity of small GTPases of this family (15). However, because Rac does not interact directly with Plex-A2 $(14,15)$, which (along with NP-1) helps form a functional receptor for Sema3A, it appears that some unknown mediator links this cytoskeleton-regulatory protein with the intracellular domain of Plex-A1 or Plex-A2.

LIM kinase, which is known in other contexts to mediate some of the effects of Rho kinase activation, appears to be directly involved in actin filament depolymerization during Sema3A-induced retraction of lamellipodia and filopodia. LIM kinase is a cytosolic serine/threonine kinase that phosphorylates and inactivates the actindepolymerizing factor cofilin. Both P21-activated kinase (PAK) and Rho kinases phosphorylate threonine508 of LIM kinase and activate the kinase. Sema3A sequentially induces phosphorylation and dephosphorylation of cofilin at growth cones of DRG neurons. A synthetic cell-permeable peptide containing a cofilin phosphorylation site has been shown to suppress Sema3A-induced growth cone collapse by inhibiting LIM kinase. Likewise, a dominant negative LIM kinase, which cannot be activated by PAK or Rho, suppresses the collapsing activity of Sema3A. Phosphorylation of cofilin by LIM kinase thus may be a critical signaling event in Sema3A-induced growth cone collapse (16).

\section{Intracellular modulation of semaphorin signaling by CRMP}

The chicken CRMP-62 molecule (also known as CRMP-2) was cloned based on its ability to activate functional responses to partially purified Sema3A in a heterologous, Xenopus oocyte-based expression system. This intracellular protein is required for Sema3A-induced growth cone collapse (17). When introduced into DRG neurons, antibodies directed against CRMP-2 block Sema3A-induced growth cone collapse without altering basal outgrowth. CRMP-2 shares homology with Caenorbabditis elegans protein UNC-33. When UNC-33 is absent, nematodes move in an uncoordinated fashion, consistent with a defect in neuronal development. Indeed, although neuronal 
cell bodies are formed normally in these mutants, axon outgrowth is aberrant, perhaps as a result of abnormalities in the form and number of microtubules (18). The phenotypes of unc-33 nematodes thus suggest that CRMP might be involved in regulation of microtubule as well as actin reorganization.

CRMPs and their related proteins, TOAD (turn on after division), Ulip (UNC-33-like protein), and DRP (dihydropyrimidinase-related protein), have been identified independently by several other groups (19). This gene family comprises at least five members in rodents (Figure 1 ), including the recently identified murine protein CRMP-5 (20) and its rat homolog, the CRMP-3-associated molecule (CRAM) (21). Most of the CRMPs are expressed predominantly in developing neurons (19), although CRMP-2 is also found in significant quantities in the lung (22). Interestingly, the C. elegans homologs of CRMPs are expressed in muscle and hypodermis cells as well as neurons, suggesting non-neuronal function of CRMPs in nematodes as well (23).

CRMP is extensively phosphorylated on serine residues $(19,24)$. The kinases involved, as well as the identity of the phosphorylated residues and the functional consequences of serine modification, are unknown, but CRMP-2 has been recently shown to be phosphorylated on threonine- 555 by the Rho-associated coil-forming protein kinase (ROCK) (also known as ROK; ref. 25). Threonine phosphorylation of CRMP-2 occurs in chick DRG during lysophosphatidic acid-induced (LPAinduced) growth cone collapse, a process that can be blocked using a dominant negative form of ROCK. Interestingly, this modification is not detected during Sema3A-induced growth cone collapse, which is insensitive to the dominant negative ROCK mutant, thus establishing that growth cone collapse can occur through ROCK-dependent and -independent pathways. Conversely, a dominant negative mutant form of Rac1 blocks Sema3A-induced but not LPA-induced growth cone collapse (12). Although CRMP-2's role in Sema3A-induced growth cone collapse is still ill-defined, these findings suggest that phosphorylation of CRMP-2 is responsible for a ligand-dependent growth cone collapse.

CRMP-2 is also associated with a dramatic morphological change in cultured hippocampal neurons (26), where increased CRMP-2 immunoreactivity is detected in the growth cone and distal parts of growing axons. In cultures transfected with CRMP-2, a majority of CRMP-2-expressing neurons bear supernumerary axons of different numbers. CRMP-2 also induces axonal sprouting from established dendrites. This effect is not seen in cells overexpressing the $\triangle$ C548-CRMP-2 mutant, which lacks the crucial ROCK recognition site required for threonine phosphorylation of CRMP-2.

\section{Semaphorin-induced growth cone collapse and axoplasmic transport}

The similarity between CRMP and UNC-33 and the microtubule phenotypes of unc-33 mutant nematodes initially suggested that Sema3A might have some effects on the axoplasmic transport system, in which microtubules are known to act. We have found that Sema3A, at a comparable dose to that required for growth cone collapse, augments antero- and retrograde axoplasmic transport of organelles (27). Sema3A induces axoplasmic transport via NP-1, the same receptor that mediates growth cone collapse. A recombinant soluble form of NP-1 has been shown to block Sema3A-induced axoplasmic transport; a functionblocking anti-NP-1 antibody has a similar effect (4). This physiological response to Sema3A appears to occur independently of growth cone collapse, since pertussis toxin, which inactivates the $\alpha_{i}$ and $\alpha_{o}$ subunits of trimeric $G$ proteins, blocks Sema3A-induced growth cone collapse (5) but does not affect Sema3Ainduced axoplasmic transport. Hence, NP-1 appears to initiate distinct signal transduction cascades, leading to growth cone collapse or facilitating axoplasmic transport (Figure 2). In the growth cone, these two NP-1-dependent responses occur simultaneously. Sema3A also accelerates endocytosis at sites of NP-1/Plex-A1-F-actin colocalization during growth cone collapse, implying that this semaphorin can alter membrane dynamics to promote vesicular trafficking, as well as actin-cytoskeletal reorganization $(4,27,28)$.

\section{Non-neuronal functions of semaphorin signaling}

Although semaphorins and their receptors are recognized for their roles in axon guidance, they are also expressed in various non-neuronal organs and tissues

\footnotetext{
Figure 2

Schematic representation of possible signal transduction pathways triggered by Sema3A. This semaphorin binds a complex containing the receptor proteins Plex-A1 and NP-1, as well as other, still poorly characterized signal transducing molecules. In neurons, some of the downstream events, including reorganization of the actin cytoskeleton and the consequent collapse of the axonal growth cone, are also influenced by pertussis toxin-sensitive (PTX-sensitive) G proteins. The CRMP appears to be central to several of the cellular responses to Sema3A.
}

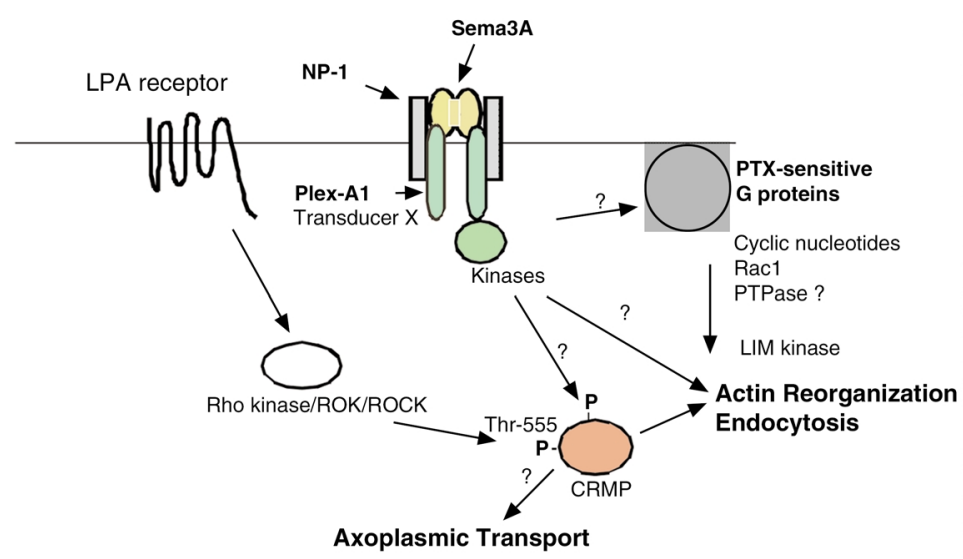


such as the heart, bone, kidney, intestine, and lung $(2-5)$. These molecules are proposed to act in lung development (22, 29), angiogenesis $(30,31)$, hematopoiesis, and endocrine regulation. Their ability to mediate cell contraction (8), adhesion, and migration $(31,32)$, as well as growth and differentiation, establishes intriguing parallels with the invasive growth phenotype discussed in the other articles in this Perspective series, especially in light of the conserved Sema domain that is found not only in semaphorins and plexins, but also in Met, a well-known inducer of invasive growth (see Comoglio and Trusolino, this series, ref. 33). In addition, one transmembrane semaphorin has been implicated in the regulation of immune function (34).

\section{CD100, a class 4 semaphorin widely expressed in the immune system}

The $150-\mathrm{kDa}$ transmembrane protein CD100/Sema4D, a member of the class 4 semaphorin subfamily, is the only semaphorin member known to be expressed by normal immunocytes, such as $\mathrm{T}$ and $\mathrm{B}$ lymphocytes, natural killer cells, monocytes, and neutrophils (35). The CD100 cytoplasmic tail carries one site for tyrosine phosphorylation and multiple sites for serine/threonine phosphorylation. In addition, CD100, like other transmembrane semaphorins, can be processed to a soluble form that may be bioactive in its own right. CD100 release appears to be mediated by metalloproteinases (36). In T cells, only soluble CD100 dimers are produced, suggesting that CD100 dimerization is required for proteolysis.

CD100-deficient mice show severe impairment of B cell development and blunted humoral immune responses (34). In addition, antibody cross-linking of CD100 provides a proliferative signal to $\mathrm{T}$ cells in the presence of submitogenic doses of anti-CD3 or antiCD2 antibodies, suggesting that CD100 is involved in $\mathrm{T}$ cell activation. This semaphorin is associated with CD45, a cell surface protein tyrosine phosphatase (PTP), which is a key molecule in T cell receptor activation. Interestingly, CD100-associated CD45 PTP activity increases upon $\mathrm{T}$ cell activation. Plex-B1 has been suggested to be a high-affinity receptor for CD100 in neuronal cells (9), but it is unknown whether it plays such a role in the immune system. Recently, CD72, a negative regulator of $\mathrm{B}$ cell responsiveness, has been identified as a putative CD100 receptor on lymphocytes (34). CD100 stimulation induces tyrosine dephosphorylation of $\mathrm{CD} 72$ and dissociation of SHP-1 from CD72.

\section{Semaphorins and their receptors in the vascular system}

NP-1 is also a receptor for a specific isoform of VEGF, VEGF $_{165}$ (37). VEGF is an important factor in the early development of the vascular system and also plays an essential role in tumor cell survival and proliferation and in angiogenesis during embryogenesis $(31,32)$. VEGF binds to two receptor tyrosine kinases, KDR/ Flk-1 and Flt-1. Coexpression of NP-1 with KDR enhances the binding of $\mathrm{VEGF}_{165}$ to $\mathrm{KDR}$ and promotes
$\mathrm{VEGF}_{165}$-mediated chemotaxis. Conversely, inhibition of VEGF $\mathrm{V}_{165}$ binding to NP-1 prevents its binding to KDR and blocks its mitogenic activity for endothelial cells. Remarkably, VEGF ${ }_{165}$ stimulates, whereas Sema3A inhibits, the motility of vascular endothelial cells expressing NP-1 (31). Furthermore, Sema3A and $\mathrm{VEGF}_{165}$ compete for NP-1 binding sites. These findings indicate convergence of two different factors, semaphorin and VEGF, on a single receptor, NP-1.

Like NP-1, NP-2 has been detected in various tumor tissues $(30,32)$ and has been proposed to promote tumor angiogenesis and progression. Complexes containing VEGF receptor-1 and one or both of the NP proteins may be part of a cell membrane-associated signaling complex for VEGF or other factors.

\section{Lung development and semaphorin signaling}

To explore a functional aspect of this system in a nonneuronal tissue, we have examined the expression of the semaphorins and their receptors in mouse lung development $(22,29)$. Lung development requires cell proliferation, migration, acquisition of cell polarity, branching morphogenesis, and differentiation to various fetal cell types. Various molecules, semaphorins among them, have been reported to work in concert to integrate the responses of epithelial cells and mesenchymal cells during lung development. Interestingly, several small cell lung cancer cell lines exhibit homozygous deletion in the chromosomal region 3 p21.3, where the genes for Sema3B and Sema3F are found (29), raising the possibility that a defect in semaphorin signaling contributes to tumor progression in this organ. A recent report showed that patients with high CRMP-1-expressing lung carcinomas exhibited lower lymph node metastasis and better prognosis, and that transfection of CRMP-1 into lung carcinoma cells induced loss of F-actin-positive filopodia and decreased invasive ability in matrigel (38). It is also notable that CRMP-2 is expressed in lung epithelial cells and neuroendocrine cells (22), further strengthening the notion that the establishment or maintenance of normal pulmonary structure requires several players usually associated with neuronal growth and morphogenesis.

Histochemical observation of adult and fetal mouse lungs revealed distinct spatiotemporal expression patterns of class 3 semaphorins and their receptors (22, 29) (Figure 3). In early pulmonary development (embryonic days 11-13), Sema3A is expressed mainly in the distal mesenchymal tissue, whereas Sema3C is expressed mainly in the large airway epithelium, and Sema3F is weakly expressed in both epithelial and mesenchymal cells. At this stage, expression of the receptors NP-1 and NP-2 is clearly coordinated with that of their ligands: NP-1 in the epithelium is preferentially localized to the terminal buds, adjacent to the mesenchyme where Sema3A is strongly expressed; NP-2 expression likewise largely overlaps with that of Sema3C. Later in gestation and after birth, expression of NP-1 is detected in distal alveolar epithelia, as well as in mesenchymal and endothelial cells. In addition, 


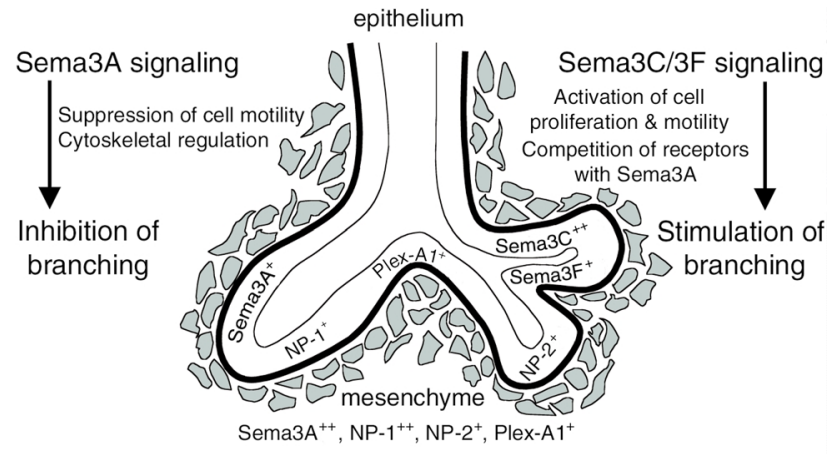

Figure 3

Model of semaphorin functions in lung development. Sema3A opposes the effects of Sema3C and Sema3F, which activate branching morphogenesis in this tissue. The expression of the semaphorins and their receptors is tightly regulated, both spatially and temporally. The expression levels of semaphorins, neuropilins, and plexins are indicated as follows: ++, high; +, moderate; (see details in Kogashima et al, ref. 29).

Sema3A is expressed in the terminal epithelial and mesenchymal tissues. Sema3C and Sema3F are also expressed in the distal developing epithelia in the middle of the lung developing period. These expression profiles suggest that the semaphorin signaling helps direct alveolar development, including the tightly apposed capillary network, consistent with evidence that semaphorins can participate in angiogenesis in normal and tumor tissues $(2,30,32)$.

By day 15, NP-2 expression is prominent in the bronchiolar epithelium and overlaps with the distribution of Sema3A. NP-2 is also expressed in mesenchymal tissues and vessels of the lung throughout the fetal period. Plex-A1, an additional component thought to form functional receptor complex with NPs for Sema3A, is diffusely expressed in epithelial and mesenchymal tissues in the early lung developmental period and is strongly expressed in the terminal epitheli$\mathrm{um}$. The Plex-A1 expression pattern is similar to that of Sema3F and NP-1.

Functional assays support the model that semaphorins contribute to lung development. Class $3 \mathrm{sem}$ aphorins show dual effects on branching morphogenesis of the cultured fetal lung tissues in early lung development $(22,29)$. Exogenous Sema3A inhibits branching morphogenesis, while Sema3C and Sema3F enhance it. These effects are probably mediated through NP-1 and NP-2 or both, since the Sema3Adependent blockade of branching morphogenesis can be overcome by providing soluble NP-1. Likewise, the effects of Sema3C and Sema3F are blocked by either soluble NP-1 or NP-2, which contain the entire extracellular domains of NP-1 and NP-2 and bind with high affinity to the corresponding semaphorins.

The mechanisms of positive or negative effects of the class 3 semaphorins on the branching morphogenesis are unclear, but it has been established that Sema3A fails to block branching morphogenesis in NP-1-deficient mice (22). Differences in the morphogenetic effects may be explained by localized expression or receptor usage, or by cell type-specific differences in proliferative or migration responses. Cross-talk or competition with other receptors may also contribute to the functional differences among semaphorins. Thus, Sema3A competes with VEGF 165 and inhibits the motility of endothelial cells (31). Sema3C could act as a competitive antagonist for Sema3A by binding to NP-1 (5). Sema3F immunoreactivity is detected in the pseudopods and in the leading edge of cultured human lung carcinoma cells, suggesting a role for this semaphorin in cell motility (32). Sema3C and Sema3F, but not Sema3A, activate cell proliferation in cultured lung epithelia by promoting progression into S-phase.

Thus the regulated expression of class 3 semaphorins and their receptors in the pulmonary epithelial and alveolar cells suggests a role for these proteins in airway development, most likely in branching morphogenesis, as is found in tissue culture studies. However, because many factors, such as FGF-10, sonic hedgehog, and retinoic acid, regulate lung branching morphogenesis, further studies are required to explore interactions between semaphorins and other signaling systems in controlling this aspect of lung development.

\section{Conclusions}

Our knowledge of the roles of semaphorins and their receptors remains far from complete, but it appears that interactions among these molecules provide a basis for finely tuned signaling and intercellular crosstalk during organogenesis. Exploration of the mechanisms by which they mediate such responses should illuminate the central processes of cell growth, migration, and gain and loss of cell polarity during development and tumorigenesis.

\section{Acknowledgments}

We would like to thank friends and colleagues for useful discussions. This work was supported by CREST of Japan Science and Technology Corp. (Y. Goshima).

1. 1999. Unified nomenclature for the semaphorins/collapsins. Semaphorin Nomenclature Committee. Cell. 97:551-552.

2. Fujisawa, H., and Kitukawa, T. 1998. Receptors for collapsin/semaphorins. Curr. Opin. Neurobiol. 8:587-592.

3. Kolodkin, A.L. 1998. Semaphorin-mediated neuronal growth cone guidance. Prog. Brain Res. 117:115-132.

4. Goshima, Y., Sasaki, Y., Nakayama, T., Ito, T., and Kimura, T. 2000. Functions of semaphorins in axon guidance and neuronal regeneration. Jpn. J. Pharmacol. 82:273-279.

5. Nakamura, F., Kalb, R.G., and Strittmatter, S.M. 2000. Molecular basis of semaphorin-mediated axon guidance. J. Neurobiol. 44:219-229.

6. He, Z., and Tessier-Lavigne, M. 1997. Neuropilin is a receptor for the axonal chemorepellent Semaphorin III. Cell. 90:739-751.

7. Comoglio, P.M., Tamagnone, L., and Boccaccio, C. 1999. Plasminogen-related growth factor and semaphorin receptors: a gene superfamily controlling invasive growth. Exp. Cell Res. 253:88-99.

8. Takahashi, T., et al. 1999. Plexin neuropilin-1 complexes form functional semaphorin-3A receptors. Cell. 99:59-69.

9. Tamagonone, L., et al. 1999. Plexins are a large family of receptors for transmembrane, secreted, and GPI-anchored semaphorins in vertebrates. Cell. 99:71-80.

10. Takahashi, T., and Strittmatter, S.M. 2001. PlexinA1 autoinhibition by the plexin sema domain. Neuron. 29:429-439.

11. Cai, H., and Reed, R.R. 1999. Cloning and characterization of neuropilin-1-interacting protein: a PSD-95/Dlg/ZO-1 domain-containing protein that interacts with the cytoplasmic domain of neuropilin-1. J. Neurosci. 19:6519-6527. 
12. Jin, Z., and Strittmatter, S.M. 1997. Rac1 mediates collapsin-1 induced growth cone collapse. J. Neurosci. 17:6256-6263.

13. Vikis, H.G., Li, W., He, Z., and Guan, K.L. 2000. The semaphorin receptor plexin-B1 specifically interacts with active Rac in a ligand-dependent manner. Proc. Natl. Acad. Sci. USA. 97:12457-12462.

14. Driessens, M.H., et al. 2001. Plexin-B semaphorin receptors interact directly with active Rac and regulate the actin cytoskeleton by activating Rho. Curr. Biol. 11:339-344.

15. Rohm, B., Ottemeyer, A., Lohrum, M., and Puschel, A.W. 2001. Plexin/neuropilin complexes mediate repulsion by the axonal guidance signal semaphorin 3A. Mech. Dev. 93:95-104.

16. Aizawa, H., et al. 2001. Phosphorylation of cofilin by LIM-kinase is necessary for semaphorin $3 \mathrm{~A}$-induced growth cone collapse. Nat. Neurosci. 4:367-373.

17. Goshima, Y., Nakamura, F., Strittmatter, P., and Strittmatter, S.M. 1995. Collapsin-induced growth cone collapse mediated by an intracellular protein related to UNC-33. Nature. 376:509-514.

18. Li, W., Herman, R.K., and Shaw, J.E. 1992. Analysis of the Caenorhabditis elegans axonal guidance and outgrowth gene unc-33. Genetics. 132:675-689.

19. Quinn, C.C., Gray, G.E., and Hockfield, S. 1999. A family of proteins implicated in axon guidance and outgrowth. J. Neurobiol. 41:158-164.

20. Fukada, M., et al. 2000. Molecular characterization of CRMP5, a novel member of the collapsin response mediator protein family. J. Biol. Chem. 275:37957-37965.

21. Inatome, R., et al. 2000. Identification of CRAM, a novel unc-33 gene family protein that associates with CRMP3 and protein-tyrosine kinase(s) in the developing rat brain. J. Biol. Chem. 275:27291-27302.

22. Ito, T., et al. 2000. Repulsive axon guidance molecule Sema3A inhibits branching morphogenesis of fetal mouse lung. Mech. Dev. 97:35-45

23. Takemoto, T., et al. 2000. Cloning and characterization of the Caenorhabditis elegans CeCRMP/DHP-1 and -2: common ancestors of CRMP and dihydropyrimidinase? Gene. 261:259-267.

24. Yoshida, H., Watanabe, A., and Ihara, Y. 1998. Collapsin response mediator protein-2 is associated with neurofibrillary tangles in Alzheimer's disease. J. Biol. Chem. 273:9761-9768.

25. Arimura, N., et al. 2000. Phosphorylation of collapsing response mediator protein-2 by Rho-kinase. Evidence for two separate signaling pathways for growth cone collapse. J. Biol. Chem. 275:23973-23980.

26. Inagaki, N., et al. 2001. CRMP-2 induces axons in cultured hippocampal neurons. Nat. Neurosci. 4:781-782.
27. Goshima, Y., et al. 1997. A novel action of collapsin: collapsin-1 increases antero- and retrograde axoplasmic transport independently of growth cone collapse. J. Neurobiol. 33:316-328.

28. Fournier, A.E., et al. 2000. Semaphorin3A enhances endocytosis at sites of receptor-F-actin colocalization during growth cone collapse. J. Cell Biol. 149:411-422.

29. Kagoshima, M., Ito, T., Kitamura, H., and Goshima, Y. 2001. Diverse gene expression and function of semaphorins in developing lung: positive and negative regulatory roles of semaphorins in lung branching morphogenesis. Genes Cells. 6:559-571.

30. Roche, J., et al. 1996. Distinct 3p21.3 deletions in lung cancer and identification of a new human semaphorin. Oncogene. 12:1289-1297.

31. Miao, H.Q., et al. 1999. Neuropilin-1 mediates collapsin-1/semaphorin III inhibition of endothelial cell motility: functional competition of collapsin-1 and vascular endothelial growth factor-165. J. Cell Biol. 146:233-242.

32. Brambilla, E., Constantin, B., Drabkin, H., and Roche, J. 2000. Semaphorin DEMA3F localization in malignant human lung and cell lines: a suggested role in cell adhesion and cell migration. Am. J. Pathol. 156:939-950.

33. Comoglio, P.M., and Trusolino, L. 2002. Invasive growth: from development to metastasis. J. Clin. Invest. 109:857-862. DOI:10.1172/JCI200215392.

34. Shi, W., et al. 2000. The class IV semaphorin CD100 plays nonredundant roles in the immune system: defective $B$ and $T$ cell activation in CD100-deficient mice. Immunity. 13:633-642.

35. Billard, C., Delaire, S., Raffoux, E., Bensussan, A., and Boumsell, L. 2000. Switch in the protein tyrosine phosphatase associated with human CD100 semaphorin at terminal B-cell differentiation stage. Blood. 95:965-972.

36. Elhabazi, A., Delaire, S., Bensussan, A., Boumsell, L., and Bismuth, G. 2001. Biological activity of soluble CD100. I. The extracellular region of CD100 is released from the surface of T lymphocytes by regulated proteolysis. J. Immunol. 166:4341-4347.

37. Soker, S., Takashima, S., Miao, H.Q., Neufeld, G., and Klagsbrun, M 1998. Neuropilin-1 is expressed by endothelial and tumor cells as an isoform specific receptor for vascular endothelial growth factor. Cell. 92:735-745.

38. Shih, J.Y., et al. 2001. Collapsin response mediator protein-1: a lung cancer invasion suppressor gene with nerve. J. Natl. Cancer Inst. 93: $1392-1400$ 